\title{
Altruistic Distributed Target Allocation for Stable Navigation in Formation of Multi-robot System
}

\author{
A. Benzerrouk ${ }^{1,3}$, L. Adouane ${ }^{1,3}$ and P. Martinet ${ }^{2,3}$ \\ ${ }^{1}$ Clermont Université, Université Blaise Pascal, LASMEA, BP 10448, 63000 Clermont-Ferrand, France \\ 2 IRRCYN, Ecole Centrale de Nantes, 1 rue de la Noé, BP 92101, 44321 Nantes Cedex 03, France \\ ${ }^{3}$ CNRS, UMR 6602, LASMEA, 63177 Aubière, France \\ Ahmed.BENZERROUK@lasmea.univ-bpclermont.fr
}

\begin{abstract}
This paper deals with reaching and keeping the formation for a group of mobile robots. A set of virtual targets (points) form a virtual structure of the same shape as the desired formation. Hence, to join and to keep this formation, each robot has only to track one of these targets. The objective of the paper is mainly to propose a cooperative strategy between the robots in order to rapidly join the virtual structure. Instead of assigning ahead one target per robot, the proposed strategy consists of making each one able to negotiate the closest target. If the latter is desired by more than one robot, it is left for the one which meets more difficulties to find an other target. Negotiation is based on a minimalist communication of relative cost coefficients between the robots. Simulation and experimental results validate the proposed contributions.
\end{abstract}

\section{INTRODUCTION}

Controlling and coordinating Multi-robot systems MRS are an attractive research subject thanks to their large application fields (spatial exploration, platooning, rescue, etc.). In this paper, we are particularly interested in the navigation in formation task with a reactive manner. Works given in the literature converge to three principle approaches: hierarchical approach, behavior based, and the virtual structure strategy.

In the first approach, one or many robots are considered as leaders while the other robots are the followers. Generally, the leader tracks a predefined trajectory while the followers track its transformed coordinates [1], [2]. This approach is simple to perform. However, it is noticed that a leader failure leads to stop the whole system. In behavior based approach [3], [4], all the robots are homogeneous. It means that perception and control are equitably distributed on the robots. This method is then much more tolerant to failure [5] than the hierarchical approach. Behavior based implies that each one has a set of weighted behaviors (basic tasks) to achieve. The resulting behavior of the group emerges from the basic ones without an explicit model of the overall cooperative behavior. However, this approach is upbraided for the way to choose the applied control to each robot. In fact, according to perception information, control system switches between behaviors (competitive approach [6]), or merges several controllers (motor schema [7]). This naturally makes hard studying the stability of the overall control.

This work is supported by the French National Research Agency (ANR) through the R-Discover project.
Virtual structure approach considers the formation as a single virtual body. The shape of the latter is the desired formation shape, and its motion is translated into the desired motion of each vehicle [8], [9]. The virtual structure is generally tackled through potential field methods [10], [11]: thus, all members of the formation track assigned nodes which move into the desired configuration. Each node applies an attractive field to the corresponding robot whereas obstacles and other robots apply repulsive field to avoid collision. The weakness of the virtual structure is that potential applications are limited especially when the formation shape needs to be frequently reconfigured.

To overcome drawbacks of these strategies, it was proposed to combine virtual structure and behavior based in [12]. The achieved task (reaching and maintaining a desired formation while avoiding collision) is divided into two basic tasks (behaviors): attraction to a dynamical target, and obstacle avoidance. These behaviors do not use potential fields which allows possible reconfiguration of the formation.

In this paper, a particular attention is given to the cooperative strategy between the robots. Hence, only the part of maintaining the formation is treated and obstacle avoidance will not be detailed. In fact, in the literature, it is noticed that the formation emerges because each robot tracks its target. These targets are assigned ahead to the robots: In [4], relative positions of the robots in the formation are based on an identification number ID. Each robot obtains then the relative position corresponding to its ID. The same strategy is followed in [13]. Even in recent works, every robot tracks a target already assigned to it [14], [15], [16] and optimizing the allocation of these targets was not studied.

This preliminary assignation allows to avoid conflicts (to not choose the same target) between the robots in a simple way. However, if these robots optimize the way of sharing the targets according to their initial positions, formation can be reached faster and some deadlock situations can be avoided. In fact, by assuming homogeneous robots (they have the same constraints (maximal velocities, accelerations, etc.)), reducing covered distances leads to reducing time to reach the formation.

In this paper, we are then interested in the target negotiation between the robots. It is proposed that each one 
negotiates, in a distributed manner, the closest target with the others in order to reduce the total distance completed and thus, time to reach the formation. If one target is desired by many robots, it is given up to the one which seems having bigger costs for the other targets. A form of altruism is then observed between the robots.

The idea of the dynamic allocation of the targets is inspired from the auction sales activity. The latter is used in the literature for the task allocation to MRS [17] such as exploration [18], visiting different locations [19], and box pushing [20]. Three main auction mechanisms were developed. Combinatorial auctions [21] treat all the possible combination of the tasks. Hence, they give optimal results. However, time computation becomes easily heavy when the number of tasks and robots increase. Moreover, this computation requires a central unit with a total knowledge of the environment. This is inconsistent with our desired distributed architecture of control. Repeated parallel auctions [22], treat each task separately of the other tasks. Auctions are repeated every time interval to test if one task can be improved if it is allocated to an other robot. In sequential mechanisms [19], each robot auctions each task individually taking into account its previous state. Therefore, there is no need to a central coordination. However, robots have to communicate their costs to determine the winner of each task.

As in [23], our algorithm is close to a combination of the two last methods since each robot wins a target or gives it up to another by computing and comparing (itself) costs of these targets. Allocation of the targets can occur every $\Delta T$ to adapt the robots to the formation changes. Only a minimalist communication is needed with the proposed algorithm.

The remainder of the paper is organized as follow: in next section (II), the task of navigation in formation is defined and the dynamic allocation of the targets algorithm is detailed. Section III reminds the proposed control law insuring that each robot joins the formation [12]. Section IV gives simulation and experimental results. Finally, conclusion and some prospects are given in section $\mathrm{V}$.

\section{NAVIGATION IN FORMATION USING VIRTUAL STRUCTURE}

\section{A. The virtual structure principle}

Before discussing cooperative strategy, the adopted virtual structure principle is reminded. Consider $N$ robots with the objective of reaching and maintaining them in a given formation. The proposed virtual structure that must be followed by the group of robots is defined as follow:

- Define one point which is called the main dynamical target (cf. Figure 1),

- Define the virtual structure to follow by defining $N_{T}$ nodes (virtual targets) to obtain the desired geometry. Each node $i$ is called a secondary target and is defined according to a specific distance $D_{i}$ and angle $\Phi_{i}$ with respect to the main target. Secondary targets defined by this way have then the same orientation $\theta_{T}$. However, each target $i$ will have its linear velocity $v_{T_{i}}$. The number of these targets $N_{T}$ must be $N_{T} \geq N$.

An exemple to get a triangular formation is given in figure 1.

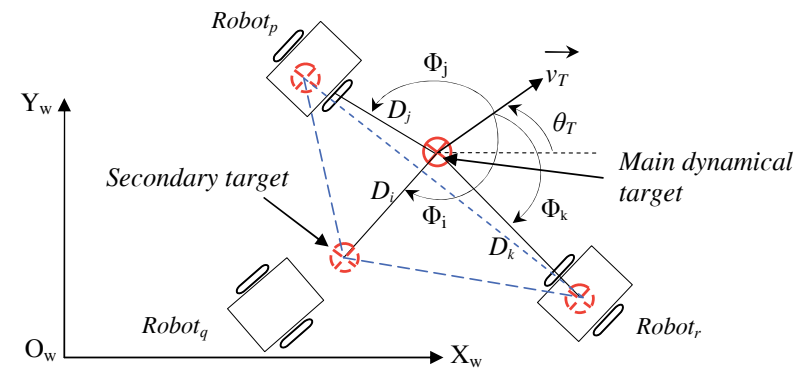

Fig. 1. Keeping a triangular formation by defining a virtual geometrical structure.

B. Cooperative strategy between the robots: dynamic allocation of the targets

The idea is that robots cooperate in order to reduce time of reaching the formation. As already discussed, each robot chooses the closest target to track. However, this may create conflicts when many robots choose the same target. To avoid this conflict, a hierarchy between them was adopted in [12]. Hence, the desired target is given up to the robot of a higher rank. However, this hierarchy is not justified especially if the robots have the same characteristics. In this paper, it is then proposed that each robot computes a coefficient per target to describe its interest for this one. Computed every time interval $\Delta T$, this coefficient informs if this target is very close or very far from the robot comparing to the other targets. It is called Relative Cost Coefficient (RCC) and is noted $\delta$. Comparing RCCs of the same target allows to each robot to decide if it takes this target or gives it up to an other.

In what follows, the RCC of a robot $i$ for the target $j$ is noted $\delta_{i j}$. It is computed as

$$
\delta_{i j}=\frac{d_{S_{i j}}}{\sum_{k=1}^{N_{T}} d_{S_{i k}}}=\frac{d_{S_{i j}}}{d_{S_{i j}}+\sum_{k=1, k \neq j}^{N_{T}} d_{S_{i k}}}
$$

where $d_{S_{i j}}$ is the distance between the robot $i$ and the target $j$. For a robot $i$, the set of RCCs for all the targets is put in a vector $\Delta_{i}$.

It is clear that (cf. Equation 1) $0 \leq \delta_{i j} \leq 1$

Moreover, $\delta_{i j}$ is as close to 0 as

$$
d_{S_{i j}} \ll \sum_{k=1, k \neq j}^{N_{T}} d_{S_{i k}}
$$

Thus, every robot prefers the target with the smallest RCC because it is the closest one. It is then noticed that the same result would be obtained by simply comparing the distances to the different targets and directly choosing the closest one. However, the main objective of the RCC is to negotiate the desired target with the others. Hence, if two robots $i$ and $k$ ask for the same target $j$ (they are in conflict for this target), 
distances $d_{S_{i j}}$ and $d_{S_{k j}}$ are not sufficient to know which robot has to obtain it in order to reach faster the formation.

Therefore, to negotiate their targets, robots act according to the following proposition:

Proposition 1. If many robots are in conflict for one target, then this target is left to the robot having the smallest RCC for this target.

In fact, according to (2), the strategy of this proposition is to compare the situation of the robots according to the existing targets and to give up the desired one to the furthest robot from the other targets. The proposed distributed strategy for dynamic allocation of the targets which allows an altruism between the robots is given in algorithm 1 .

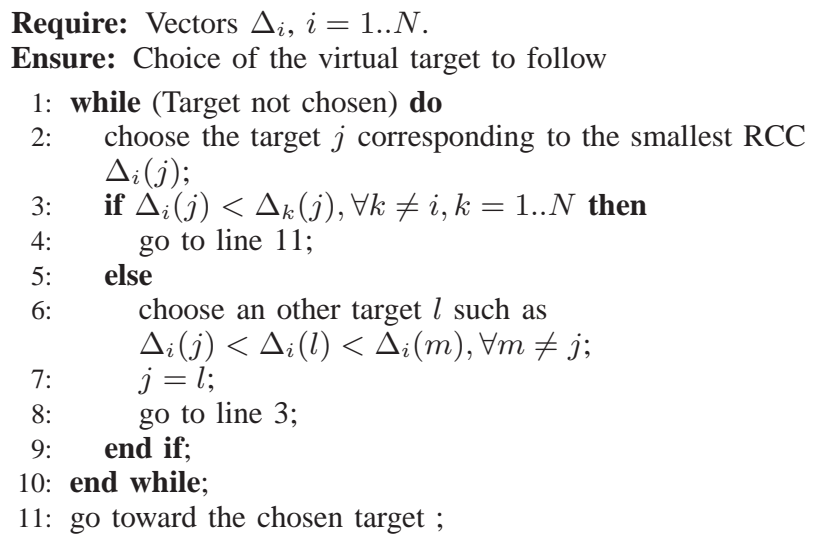

Algorithm 1: Distributed virtual target assignment $\left(N_{T} \geq N\right)$.

The proposed algorithm is distributed on all the robots. It requires that each robot $i$ communicates only its vector $\Delta_{i}$ to the other ones. It is also proposed that a vector $\Delta_{i}$ includes the subscript $i$ indicating the robot identifier. Identifiers of the robots are randomly chosen and do not indicate any hierarchy for the target assignment.

According to this algorithm, every robot is able to deduce if the desired target will be really available or it will be taken by an other one having a less corresponding RCC. Negotiation and allocation of the target is then done in a distributed manner.

It is noticed that the required communication process is very basic and can be summarized in algorithm 2 . The time interval $\delta_{t}$ to wait (line 2, algorithm 2) allows to avoid collision between network packets.

1: receive the vectors $\Delta_{k}$ such that $k=1 . . i-1$;

2: wait a time $\delta t$, then send the vector $\Delta_{i}$;

3: receive the vectors $\Delta_{k}$ such that $k=i+1 . . N$;

Algorithm 2: Sequential communication process of the robot $i$ with the other robots.

Even if most of conflicts in target assignment are solved thanks to algorithm 1, some points need to be discussed:

- if the robot $i$ has the same RCC value for a target $j$ as an other robot $k$ (which means $\Delta_{i}(j)=\Delta_{k}(j)$ ), then $i$ can search the second possible target $l$ for itself such that $\Delta_{i}(j)<\Delta_{i}(l)<\Delta_{i}(m), \forall m \neq l$, and the second possible target $n$ for the robot $k$ with $\Delta_{k}(j)<\Delta_{k}(n)<\Delta_{k}(m), \forall m \neq n$ (robot $k$ has naturally the same reasoning). The robot $i$ keeps the target $j$ if $\left(\Delta_{i}(l)>\Delta_{k}(n)\right)$, because it means that $k$ will find the target $n$ with a cheaper RCC. Otherwise, if $\left(\Delta_{i}(l)<\Delta_{k}(n)\right)$, the robot $i$ gives up the target $j$ because $l$ seems cheaper for it than the target $n$ for the robot $k$.

- If the robots $i$ and $k$ have also the same RCC for their next targets $\left(\Delta_{i}(l)=\Delta_{k}(n)\right)$ (targets $l$ and $n$ are as defined above). In this case, the target $j$ can be indifferently taken by $i$ or $k$. However, to avoid that both the robot choose the target $j$, or both leave it, it is proposed that the robot with the higher subscript obtains it. This convention cannot be considered as a hierarchy between the robots since they choose their targets with the same RCCs.

- Finally, this distributed reasoning can be easily applied if more than two robots negotiate the same target. Note that according to algorithm 2, communication is done once at the beginning of negotiations. It is then not affected (by becoming tedious) when many robots are in conflict for a target.

\section{The Applied Control to the Robots}

\section{A. Attraction to a Dynamical Target Controller}

To remind the attraction to a Dynamical Target Controller which allows to keep the formation, consider a robot $i$ with $\left(x_{i}, y_{i}, \theta_{i}\right)$ pose. This robot has to track its secondary dynamical target. To simplify notations in the following, the same subscript of the robot is given to its target. The latter is then noted $T_{i}\left(x_{T_{i}}, y_{T_{i}}, \theta_{T}\right)$ (cf. Figure 2 ) and the variation of its position can be described by

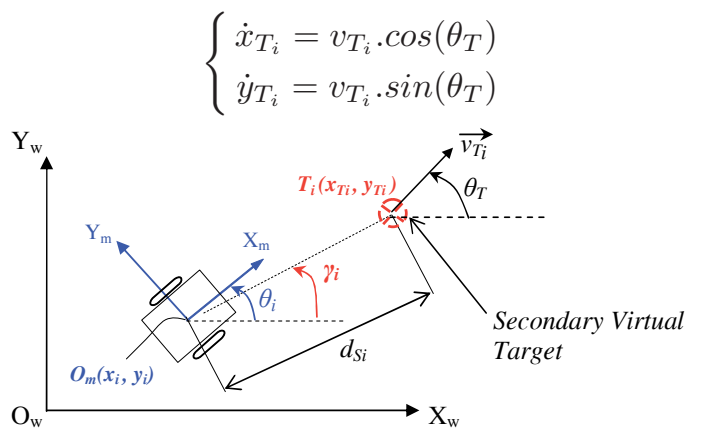

Fig. 2. Attraction to a dynamical target.

Let's also introduce the used robot model (cf. Figure 2). Experimental results are made on Khepera robots, which are unicycle mobile robots. Their kinematic model can be described by the well-known equations (cf. Equation 4).

$$
\left\{\begin{array}{l}
\dot{x}_{i}=v_{i} \cdot \cos \left(\theta_{i}\right) \\
\dot{y}_{i}=v_{i} \cdot \sin \left(\theta_{i}\right) \\
\dot{\theta}_{i}=\omega_{i}
\end{array}\right.
$$


where $\theta_{i}, v_{i}$ and $\omega_{i}$ are respectively the robot orientation, the linear and angular velocities.

The set-point angle that the robot must follow, to reach its dynamical target, is given by

$$
\theta_{S_{a t i}}=\arcsin \left(b \sin \left(\theta_{T}-\gamma_{i}\right)\right)+\gamma_{i}
$$

Where $b=\frac{v_{T_{i}}}{v_{i}} \cdot \gamma_{i}$ is the angle that the robot would have if it was directed to its target (cf. Figure 2). This set-point has been obtained by keeping $\gamma_{i}$ constant. More details and proofs are available in [12].

\section{B. The used control law}

The used control law, which was proposed in [12], allows to each robot $i$ to converge to its set-point.

$$
\begin{gathered}
v_{i}=v_{\max }-\left(v_{\max }-v_{T_{i}}\right) e^{-\left(d_{S_{i}}^{2} / \sigma^{2}\right)} \\
\omega_{i}=\omega_{S_{a t i}}+k \tilde{\theta}_{i}
\end{gathered}
$$

where

- $v_{i}$ and $\omega_{i}$ are linear and angular velocities of the robot respectively. It is also noted $\omega_{S_{i}}=\dot{\theta}_{S_{a t i}}$ where $\dot{\theta}_{S_{a t i}}$ is the angular variation of $\theta_{S_{a t i}}$.

- $v_{\max }$ is the maximum linear speed of the robot,

- $\sigma, k$ are positive constants,

- $\tilde{\theta}_{i}$ is the error orientation so that $\tilde{\theta}_{i}=\theta_{S_{a t i}}-\theta_{i}$ which gives $\dot{\tilde{\theta}}_{i}=\omega_{S_{a t i}}-\omega_{i}$.

Lyapunov based stability allowed to prove the convergence of the robot to its target [12]. Convergence of the whole multi-robot system to the set-point virtual structure can then be derived by studying the following Lyapunov function

$$
V=\sum_{k=1}^{N} V_{k}
$$

where $V_{k}$ is the Lyapunov function associated to the robot $k$. This function was defined as

$$
V_{k}=\frac{1}{2} \tilde{\theta}_{k}^{2}
$$

It has been proved that $\dot{V}_{k}<0$ (when $\tilde{\theta}_{k} \neq 0$ )[12]. Therefore, it can be easily deduced that

$$
\dot{V}=\sum_{k=1}^{N} \dot{V}_{k}<0
$$

The global system is then asymptotically stable. Moreover, it can be noticed that the applied angular velocity $\omega_{i}$ allows exponential convergence of the error orientation to 0 .

However, this theoretical convergence is applied to nonholonomic mobile robots. It means that stability will be insured only if the angular set-point is reachable by the robot while considering its kinematic constraints (maximal velocities, maximal accelerations).

In [12], it has been proved that the robot converges to its target only if

$$
v_{i} \geq v_{T_{i}} \quad \Leftrightarrow \quad b \leq 1
$$

According to equation (6a), it is noticed that the linear velocity of the robot verifies the condition given by inequation (10), and takes into account its maximal linear velocity. However, it is noted that linear velocity of the secondary targets depends on their relative position in the virtual structure. The choice of $D_{i}$ and angle $\Phi_{i}$ affect then $v_{T_{i}}$.

Moreover, the variation of the angular set-point $\dot{\theta}_{S_{a t i}}$ has to stay reachable by the robot. Indeed, the angular velocity supported by the robot has a maximal value noted $\omega_{\max }$. Defining the bounaries of $\dot{\theta}_{S_{a t i}}$ so that $\left|\omega_{i}\right| \leq \omega_{\max }$ and those of $D_{i}$ and $\Phi_{i}$ so that $v_{T_{i}}<v_{\max }$ will be discussed in a future work.

\section{Simulation And ExPERIMENTAL RESUlTs}

To show the relevance of the proposed algorithm for the dynamic allocation of the targets, it is proposed to simulate a group of 5 robots reaching a formation $(N=5)$. The MRS is simulated with different initial positions (IPs).

For every IP, the simulation is made twice: one with a prior assignation of the targets (target $T_{i}$ to robot $R_{i}$ ), and one with the proposed algorithm where the robots use the RCC to obtain their targets. In the two cases, time to reach the formation is measured in order to evaluate the proposed algorithm performance. Note that the formation is considered reached if the distance $d_{S_{i j}}$ separating every robot $R_{i}$ from the chosen target $T_{j}$ is such that $d_{S_{i j}} \leq r_{0}$; where $r_{0}$ is the radius of a small virtual circle in the neighborhood of the targets. Results are given in table I.

Comparing the two approaches, it can be seen that negotiation of the targets using RCC offers a smaller time of convergence. It is noticed that the amount of enhancement depends on the initial position of the robots. In fact, for a prior assignation case (without negotiation), robots may be in the other side of the virtual structure compared to their assigned targets. They have then to avoid each other and to uselessly navigate to far targets. To illustrate this problem, An example of initial positions is given in figure 3(a). In the case of prior assignation, robot $R_{i}$ has to join target $T_{i}$. To keep obvious the order of the targets in the figures, a straight trajectory is given to the virtual structure (see figure 3(b)) (a circular trajectory is used in experimental results). Taking the exemple of robot $R_{5}$, it can be seen that it has to go until target $T_{5}$ when it does not negotiate the closest one (cf. Figure 3(b)). However, by using RCC algorithm (cf. Figure 3(c)), it obtains the target $T_{2}$ which is much closer. Meanwhile, $R_{5}$ did not choose $T_{3}$ even if it was the closest one (cf. Figure 3(a)). In fact, it gives up $T_{3}$ to the robot $R_{3}$ which was behind it at the beginning of the simulation. In the same manner, $R_{1}$ gives up the closest target $\left(T_{5}\right)$ to $R_{4}$ and takes $T_{1}$.

TABLE I

TIME TO REACH THE FORMATION FOR DIFFERENT INITIAL POSITIONS IPS [S].

\begin{tabular}{c|c|c|} 
& Without target negotiation & with target negotiation \\
\hline$I P s_{1}$ & 9.9 & 8.2 \\
\hline$I P s_{2}$ & 12.2 & 9.3 \\
\hline$I P s_{3}$ & 20.8 & 15.9 \\
\hline$I P s_{4}$ & 21.8 & 20
\end{tabular}




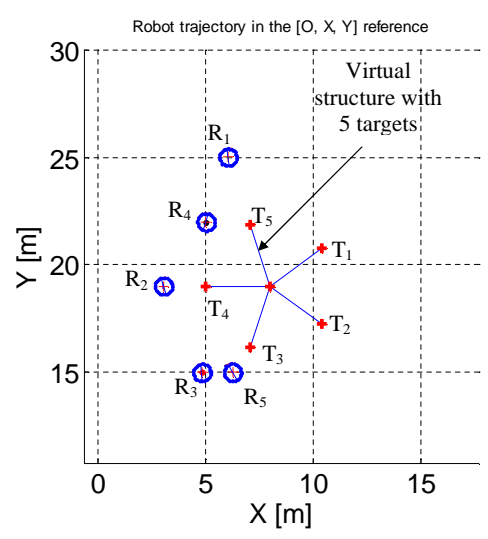

(a) Initial position of the robots

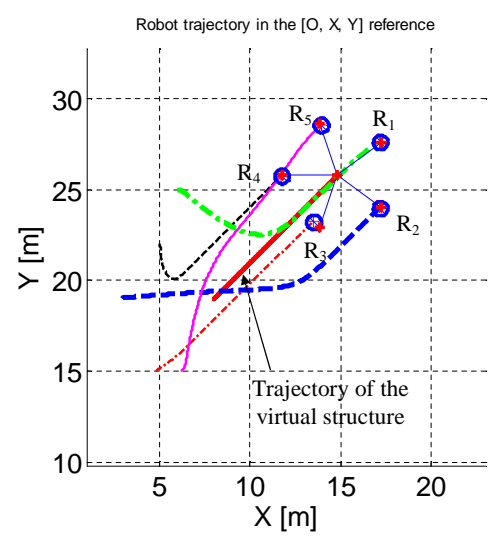

(b) A prior assignation of the targets

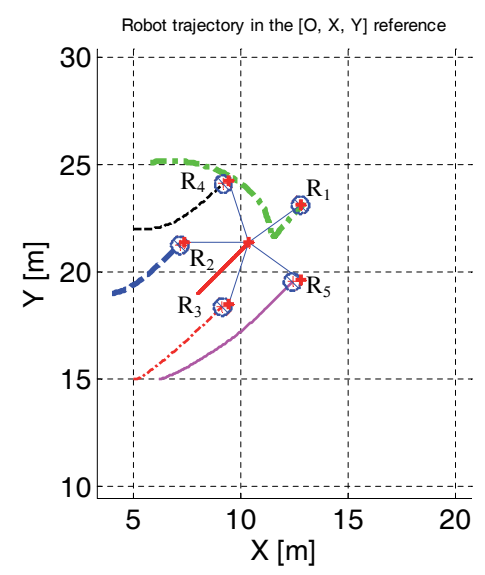

(c) Negotiation of the targets using RCC algorithm

Fig. 3. Trajectory of the robots reaching the formation: prior assignation versus negotiation of the targets.

\section{A. Experimental results: a formation of 3 robots}

Experimentations are implemented on Khepera III robots. As first tests, only perception of the MRS is still centralized. Hence, navigation is achieved on a platform equipped with a camera giving positions and orientations of the robots (cf. Figure 4). These one have to join and to maintain a triangular virtual structure. The latter has a circular trajectory such that it stays reachable. First, it has a clockwise motion (cf. Figure 5(a)).Every robot calculates then the RCC for the targets.

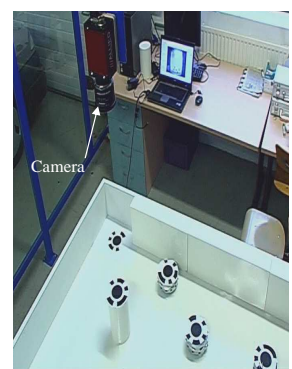

Fig. 4. Platform experimentations.

Results are given in table II.

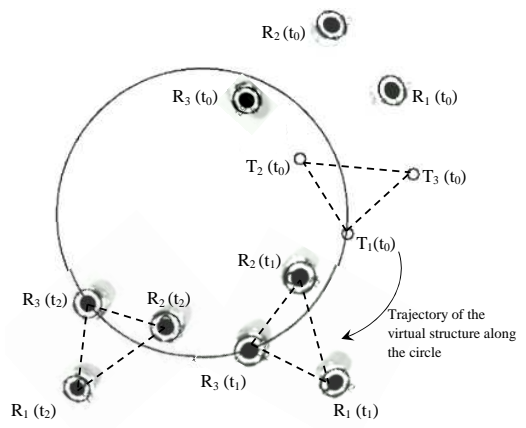
(a) $t_{0} \rightarrow t_{2}$ : clockwise motion of the virtual
structure

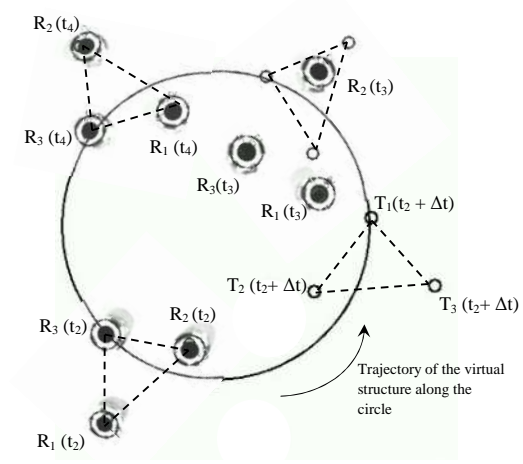

(b) $t_{2} \rightarrow t_{4}$ : switching to counter-clockwise motion

Fig. 5. Real trajectory of the robots. Distributed allocation (a) and reallocation (b) of the targets. Notation: $T_{i}\left(t_{j}\right)$ Target $i$ at moment $j, R_{i}\left(t_{j}\right)$ Robot $i$ at moment $j$.

For the robot $R_{1}$, the smallest RCC corresponds to $T_{3}$. This one is not desired by any other robot since the RCC of $R_{2}$ and $R_{3}$ for this target is not the smallest one comparing to the other targets. However, $R_{2}$ and $R_{3}$ ask both for $T_{2}$ through their RCC. Since $R_{2}$ has the smallest one, $R_{3}$ has to search for an other. It takes the remained target $T_{1}$.

TABLE II

RELATIVE COST COEFFICIENT AT MOMENT $\left(t_{0}\right)$.

\begin{tabular}{c|c|c|c} 
& $T_{1}$ & $T_{2}$ & $T_{3}$ \\
\hline$R_{1}$ & 0.41 & 0.32 & 0.25 \\
\hline$R_{2}$ & 0.39 & 0.23 & 0.33 \\
\hline$R_{3}$ & 0.39 & 0.24 & 0.41
\end{tabular}

At moment $t_{2}+\Delta t$, a jump of the virtual structure state is produced (cf. Figure 5(b)). Also, the dynamic of the virtual structure is changed so that its motion becomes counterclockwise. The robots recalculate the RCC for each target. 
The RCC are given in table III. This table shows that all the robots prefer target $T_{2} . R_{1}$ obtains it because it has the smallest corresponding RCC. $R_{2}$ and $R_{3}$ search then for the target with the RCC immediately higher than the RCC of $T_{2}$. Again, both are interested by $T_{1}$. The latter is obtained by $R_{3}$ because its RCC is smaller. $R_{2}$ takes the remained target $T_{3}$. It can be seen that $R_{2}$ and $R_{3}$ give up $T_{2}$ to $R_{1}$ (altruism). Distances between the robots and their targets are given in figure 6. They decrease until 0 which confirm that the formation is reached and maintained. When the virtual structure dynamic is changed, robots are far from their targets which explain the observed jumps. The same observations are noticed on the global Lyapunov function (cf. Figure 7).

TABLE III

RELATIVE Cost COEFFiCIENT AT MOMENT $\left(t_{2}+\Delta t\right)$.

\begin{tabular}{c|c|c|c} 
& $T_{1}$ & $T_{2}$ & $T_{3}$ \\
\hline$R_{1}$ & 0.36 & 0.21 & 0.38 \\
\hline$R_{2}$ & 0.37 & 0.22 & 0.40 \\
\hline$R_{3}$ & 0.34 & 0.26 & 0.40
\end{tabular}
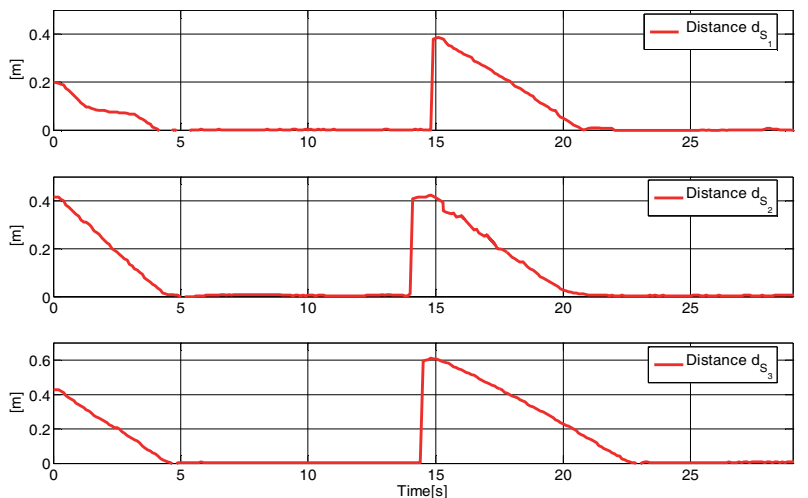

Fig. 6. Variation of the distance $d_{S_{i}}$ between the robot $i$ and the chosen target $(i=1 . .3)$.

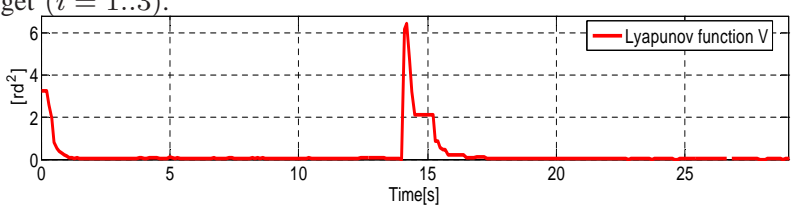

Fig. 7. Evolution of the global Lyapunov function $V$.

\section{CONCLUSIONS AND FUTURE WORKS}

In this paper, the formation of a multi-robot system, based on the virtual structure strategy, was studied. A cooperative protocol between the robots was proposed in order to rapidly join the formation. Instead of a prior assignation of their places in the formation (virtual targets), it is proposed that each robot negotiates its one with the others by communicating relative cost coefficients for each target. An altruism is then observed between the robots. Moreover, simulation results show the performance offered by the proposed algorithm. Time to reach the formation was improved by reducing trajectory of the robots. Even if the obtained results are not the most optimal ones, this work addresses an interesting subject and allows to improve this time by discussing the RCC formula.

In the proposed experimental results, dynamical negotiation of the targets was enabled at the beginning of the experimentation and when switching to the virtual structure dynamic. In order to be done in a completely reactive way, discussing and justifying its frequency $(\Delta T)$ will be the subject of future works. Collision avoidance between the robots and with other dynamical obstacles will be detailed in a future paper.

\section{REFERENCES}

[1] N. Léchevin, C. Rabbath, and P. Sicard. Trajectory tracking of leaderfollower formations characterized by constant line-of-sight angles. Automatica, 42:2131-2141, 2006.

[2] T. Gustavi and X. Hu. Observer-based leader-following formation control using onboard sensor information. IEEE Transactions on Robotics, 24:1457-1462, 2008.

[3] G. Antonelli, F. Arrichiello, and S. Chiaverini. The nsb control: a behavior-based approach for multi-robot systems. PALADYN Journal of Behavioral Robotics, 1:48-56, 2010.

[4] T. Balch and R.C. Arkin. Behavior-based formation control for multirobot teams. IEEE Transactions on Robotics and Autmation, 1999.

[5] L.E. Parker. On the design of behavior-based multi-robot teams. Journal of Advanced Robotics, 10:547-578, 1996.

[6] R. A. Brooks. A robust layered control system for a mobile robot. IEEE Journal of Robotics and Automation, 2:14-23, 1985.

[7] R. C. Arkin. Motor schema-based mobile robot navigation. International Journal of Robotics Research, 8(4):92-112, 1986.

[8] K. D. Do. Formation tracking control of unicycle-type mobile robots In IEEE International Conference on Robotics and Automation, pages 527-538, 2007.

[9] X. Li, J. Xiao, and Z. Cai. Backstepping based multiple mobile robots formation control. In IEEE International Conference on Intelligent Robots and Systems, pages 887 - 892, 2005.

[10] P. Ogren, E. Fiorelli, and Leonard N. E. Formations with a mission: Stable coordination of vehicle group maneuvers. In 15th International Symposium on Mathematical Theory of Networks and Systems, 2002.

[11] S. Mastellone, D.M. Stipanovic, and M.W. Spong. Remote formation control and collision avoidance for multi-agent nonholonomic systems. In IEEE International Conference on Robotics and Automation, pages 1062-1067, 2007.

[12] A. Benzerrouk, L. Adouane, L. Lequievre, and P. Martinet. Navigation of multi-robot formation in unstructured environment using dynamical virtual structures. IEEE/RSJ International Conference on Intelligent Robots and Systems, 2010.

[13] A. Lewis and K-H. Tan. High precision formation control of mobile robots using virtual structures. Autonomous Robots, 4:387-403, 1997.

[14] J. Ghommam, M. Saad, and F. Mnif. Formation path following control of unicycle-type mobile robots. IEEE International Conference on Robotics and Automation, pages 1966 - 1972, 2008.

[15] E. Lalish, K.A. Morgansen, and T. Tsukamaki. Formation tracking control using virtual structures and deconfliction. IEEE Conference on Decision and Control, 2006.

[16] W. Ren and R.W. Beard. Formation feedback control for multiple spacecraft via virtual structures. IEEE Proceedings on Control Theory and Applications, 151:357 - 368, 2004.

[17] M.B. Dias, R. Zlot, N. Kalra, and A. Stentz. Market-based multi-robot coordination: a survey and analysis. In Proceedings of the IEEE 94 (7), pages 1257-1270, 2006.

[18] N. Kalra, D. Ferguson, and A. Stentz. Hoplites: a market-based framework for planned tight coordination in multirobot teams. International Conference on Robotics and Automation, pages 1170-1177, 2005.

[19] C. Tovey, M. Lagoudakis, S. Jain, and S. Koenig. The generation of bidding rules for auction-based robot coordination. In Multi-Robot Systems Workshops, pages 3-14, 2005

[20] P.G. Brian and M. J. Mataric'. Sold!: Auction methods for multirobot coordination. IEEE Transactions on Robotics and Automation, 18:758-768, 2002.

[21] M. Berhault, H. Huang, P. Keskinocak, S. Koenig, W. Elmaghraby, P. Griffin, and A. Kleywegt. Robot exploration with combinatorial auctions. IEEE/RSJ International Conference on Intelligent Robots and Systems, pages 1957-1962, 2003.

[22] M. B. Dias. TraderBots: a market-based approach for resource, role, and task allocation in multi-robot coordination. $\mathrm{PhD}$ thesis, CarnegieMellon university, 2004.

[23] M. Nanjanath and M. Gini. Repeated auctions for robust task execution by a robot team, 2010 . 